Conclusions The utilisations of financial aid, counselling, and access for medical care services that provided by NPO in Taiwan were significantly with the health related variables which included the depression, adherence, CD4, and quality of life. The result could provide suggestions about refining the contents of services and promoting their function and the quality of life of PLWHA.

\section{LBP-1.10 MISCLASSIFICATION OF SYPHILIS CASES USING A REACTIVE ENZYME IMIMUNOASSAY AND REACTIVE RPR ALGORITHM ALONE FOR DIAGNOSIS}

\section{doi:10.1136/sextrans-2011-050119.16}

${ }^{1} \mathrm{~A}$ Singh, ${ }^{2} \mathrm{~K}$ Fonseca, ${ }^{3} \mathrm{~S}$ Mukhi, ${ }^{1} \mathrm{~J}$ Gratrix, ${ }^{3} \mathrm{~S}$ Plitt, ${ }^{1} \mathrm{R}$ Read, ${ }^{1} \mathrm{~K}$ Sutherland, ${ }^{2} \mathrm{G}$ Zahariadis, ${ }^{2} \mathrm{G}$ Tyrrell, ${ }^{4} \mathrm{~B}$ Lee. ${ }^{1}$ Alberta Health Services, Edmonton, Canada; ${ }^{2}$ Alberta Provincial Laboratory for Public Health, Canada; ${ }^{3}$ Public Health Agency of Canada, Canada; ${ }^{4}$ University of Alberta, Canada

Background Recent recommendations propose that samples dually reactive by a syphilis enzyme immunoassay (sEIA) and RPR be reported as positive for syphilis without confirmatory testing Methods Samples from 1 September 2007 to 19 March 2011 testing reactive by sEIA and tested by INNO-LIA (IL) were extracted from the Alberta Provincial Laboratory for Public Health's DIAL (Data Integration for Alberta Laboratories), a web based application. Syphilis testing history was reviewed for all patients with a reactive sEIA and reactive RPR and negative (NEG) or indeterminate (IND) result by IL. Syphilis infection was defined by a positive confirmatory test (majority IL; a few TPPA and FTA-ABS). The significance of RPR titres in patients NEG/IND by IL with or without evidence of syphilis infection was analysed using $\chi^{2}$ test. Median standard cut-offs (s/co) for the sEIA were compared using the MannWhitney U test.

Results 6195 samples from 4695 patients with reactive sEIA were also tested by IL: 15 samples $(0.2 \%)$ had no reported RPR result, $4232(48.3 \%)$ were non-reactive by RPR, and 1948 (31.4\%) samples from 1753 patients were reactive by RPR. 72 (4.1\%) of the 1753 patients with reactive RPR had at least one specimen tested NEG/ IND by IL (Abstract LBP-1.10 figure 1). 3 of the 72 patients (4.2\%) had a serological history of syphilis infection not recognised initially, 15 (20.8\%) had no follow-up testing, 23 (31.9\%) had a subsequent positive IL, and 31 (43.1\%) did not demonstrate syphilis infection on follow-up testing. For the 31 patients with no serological evidence of syphilis infection, 24 remained NEG/IND by IL and seven tested negative by sEIA on follow-up. Overall, 31 patients (1.7\%) would have been misclassified as infected based upon an algorithm of dually reactive sEIA and RPR without confirmatory testing. $14.7 \%$ of samples that tested negative or IND for IL in confirmed syphilis had RPR titres $=1: 8$ as compared to $20.0 \%$ of samples from patients without syphilis on follow-up serology $(p=0.5)$. The median $s / c o$ of the screening sEIA for samples from the patients who were infected

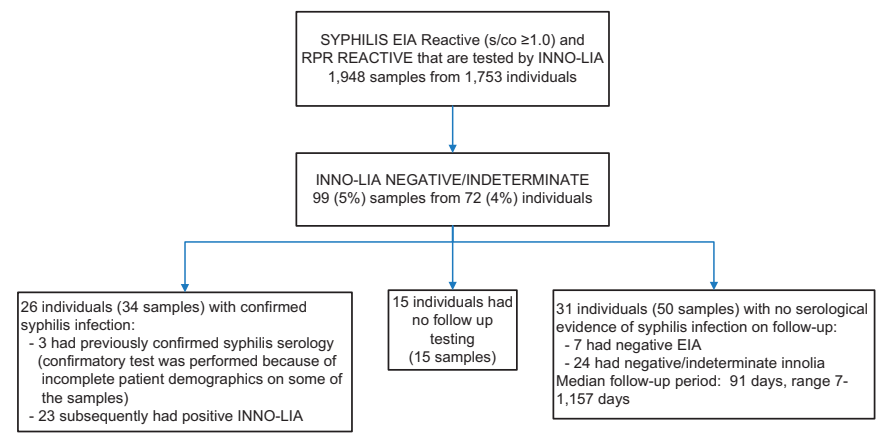

Abstract LBP-1.10 Figure 1 Misclassification syphilis cases
(4.8, range: $1.0-24.20)$ differed from the s/co (2.6, range: $1.0-12.4)$ for those uninfected $(p<0.05)$.

Conclusions In our experience, 1.7\% (31 patients) would have been misclassified as a case of syphilis if a third confirmatory test for syphilis had not been conducted. Additional evaluation of syphilis testing algorithms is warranted before a two test algorithm is widely employed.

\section{LBP-1.11 ACCEPTABILITY OF SHORT-COURSE AZT PREVENTION REGIMEN BY HIV INFECTED PREGNANT WOMEN; SHOULD VCT IN THE ANTENATAL SETTING BE MODIFIED}

doi:10.1136/sextrans-2011-050119.17

${ }^{1}$ S Abdulah Aziz, ${ }^{1} \mathrm{D}$ Mubiru, ${ }^{2} \mathrm{~N}$ Rose. ${ }^{1}$ Positive Men Union Uganda, Kampala, Uganda; ${ }^{2}$ Entebbe Infectious Diseases Institute

Background Acceptability VCT by pregnant women is critical in the contest of trials accessing interventions to reduce mother to- child transmission of HIV. We studied the logistics and uptake of shortcourse oral AZT regimen by HIV-infected women after VCT in areas of Uganda.

Methods From June 2004, a pilot project on the feasibility of shortcourse AZT was launched in an antenatal clinic in mulago hospital All pregnant women hear a 15- min talk by clinic nurses a bout mother to- child transmission of HIV and are offered voluntary preand post-test counselling by lay community volunteers. Consenting HIV-positive women are offered AZT (300 mg twice daily) from 36 weeks gestation until labour, one tablet at onset of labour, and then every $3 \mathrm{~h}$ delivery. HIV positive women are counselled and supported on their choice of infant.

Results Over a 6-month period, 1062 antenatal women were offered VCT, 247 (22\%) underwent pre-test counselling and $206(18 \%)$ agreed to be tested. Among those tested 78 (38\%) were HIV-positive, of these $17(83 \%)$ returned to collect results, including 65 HIV positive women. As of September 2004, 40 (62\%) women consented for AZT, 17 women have completed the regimen, 5 are currently receiving drug, 7 are eligible to start AZT and 11 women dropped out of the study (preterm births, incorrect dates, failure to notify nurses during labour, and non-compliances). Of the 17 women who received AZT, 12 opted for formula feeding and five women chose breast feeding.

Conclusion HIV prevalence in this setting is estimated at $30 \%$ of 332 projected HIV infected women seen, only $40(12 \%)$ women actually received AZT. The major barrier appears to be entrance into counselling. When counselled, most HIV-pregnant women choose to receive AZT prophylaxis. New approaches to antenatal HIV counselling and testing are urgently required to improve future acceptance of VCT and the successful implementation of antiretroviral pro-phylaxis.

\section{LBP-1.12 HIV AND HEPATITIS C PREVALENCE IN INDIVIDUALS LEAVING PRISON AND ENTERING DRUG AND ALCOHOL SERVICES IN THE AREA OF HIGHEST HIV PREVALENCE IN THE UK}

doi:10.1136/sextrans-2011-050119.18

H Williams, D Axten, F Makia, A Teague, J Fox. Guy's and St Thomas' NHS Foundation Trust, London, UK

Background Intravenous drug users, particularly those in prison, are at high risk of acquiring HIV and Hepatitis $\mathrm{C}(\mathrm{HCV})$ and commonly do not access mainstream medical care. Missed opportunities for HIV and Hepatitis C testing in Intravenous drug users attending prisons are therefore common. By using oral swabs difficulties of venous access can be avoided. Lambeth has the highest prevalence of 